\title{
System-Pharmacology Dissection of Traditional Chinese herbs SINI Decoction for Treatment of Cardiovascular Diseases
}

\author{
ZHIMING SHU ${ }^{1 *}$, TIANTIAN WU ${ }^{1 *}$, MOHAMED SHAHEN ${ }^{12^{*}}$, ZIHU GUO ${ }^{1}$, JIA SHU ${ }^{1}$, HE WANG ${ }^{1}$, AKHTAR H. \\ SHAR ${ }^{1}$, MAYADA R. FARAG ${ }^{3}$, MAHMOUD ALAGAWANY ${ }^{4 *}$, MOHAMED E. ABD EL-HACK ${ }^{4}$ and CHAOBIN LIU ${ }^{5 *}$ \\ ${ }^{1}$ College of Life Science, Northwest A\&F University, 712100, Shaanxi, Yangling, China \\ ${ }^{2}$ Zoology Department, Faculty of Science, Tanta University, 31527 Tanta, Egypt \\ ${ }^{3}$ Forensic Medicine and Toxicology Department, Veterinary Medicine Faculty, Zagazig University, 44511, Zagazig, Egypt \\ ${ }^{4}$ Department of Poultry, Faculty of Agriculture, Zagazig University, 44511, Zagazig, Egypt \\ ${ }^{5}$ College of Forestry, Northwest A\&F University, 712100, Shaanxi, Yangling, China
}

Manuscript received on May 7, 2018; accepted for publication on June 19, 2018

How to cite: SHU Z, WU T, SHAHEN M, GUO Z, SHU J, WANG H, SHAR AH, FARAG MR, ALAGAWANY M, ELHACK MEA AND LIU C. 2019. System-Pharmacology Dissection of Traditional Chinese herbs SINI Decoction for Treatment of Cardiovascular Diseases. An Acad Bras Cienc 91: e20180424. DOI 10.1590/0001-3765201920180424.

\begin{abstract}
Cardiovascular diseases (CVDs) are leading causes of death in the world, owing to noticeable incidence and mortality. Traditional Chinese Medicine (TCM) SINI Decoction (SND) is used to prevent and treat CVDs, which has attracted extensive attention for its moderate and little side effects. However, the involved molecular mechanisms are exceedingly complicated and remain unclear. Systems pharmacology, as a novel approach that integrates systems biology and pharmacology plays a significant role in investigating the molecular mechanism of TCM. In systems pharmacology approach, we use to systematically uncover the mechanisms of action in Chinese medicinal formula SND as an effective treatment for CVDs, which mainly includes:1) molecular database building; 2) ADME evaluation; 3) target-fishing 4) network construction and analysis. The results show that 78 underlying valid ingredients and their corresponding 71 direct targets of SND were obtained. And SND take part in cardiomyocyte protection, blood pressure regulation, and lipid regulation module in treatment of CVDs by cooperative way. Systems pharmacology as an emerging field that investigates the molecular mechanisms of TCM through pharmacokinetic evaluation target prediction, and pathway analysis, which will facilitate the development of traditional Chinese herbs in modern medicine.
\end{abstract}

Key words: systems pharmacology, SINI Decoction, Cardiovascular Diseases, molecular mechanisms, traditional Chinese herbs.

Correspondence to: Mahmoud Alagawany

mmalagwany@zu.edu.eg

ORCid: https://orcid.org/0000-0002-8020-0971

Chaobin Liu

liuchaobin@126.com

https://orcid.org/0000-0003-1827-6235

*These authors contributed equally to this work 


\section{INTRODUCTION}

The cardiovascular diseases (CVDs) are major causes of death universally. In 2012, about 17.5 million peoples died from CVDs representing about $31 \%$ of all deaths around the world and the death rates would be estimated to increase 23.6 million by 2030 . In recent years, there are many different drugs have been used to treat CVDs, mainly including antianginal drugs, anti-arrhythmic drugs, anti-coagulant medicine, blood-lipid lowering drugs, anti-hypertensive agents, beta-blockers, calcium blockers, cardiac glycosides, hydragog and vasodilator agents (Khand et al. 2000, Shu et al. 2018). These drugs are mostly playing vital roles in the single-compound and single-target, which bring many serious side effects, such as postural hyperpiesia, depressed and feeble from beta-blockers, sychnosphygmia, headache, facial flushing and polyuria from calcium blockers (Russell 1988, Yatoo et al. 2017). However, the Traditional Chinese Medicines (TCMs) have drawn great attention in the world because of little side effects and playing therapeutic role by multi-compounds and multi-targets with have coordination ( $\mathrm{Su}$ et al. 2008, Zhang et al. 2019). The Sini Decoction (SND) is one of the most important TCM recipes on CVDs which comprises three herbs: Aconiti Lateralis Radix Praeparata (ALRP, Chinese name Fuzi), Zingiberis Rhizoma (ZR, Chinese name Ganjiang), and Glycyrrhizae Radix (GR, Chinese name Gancao) at a ratio of $3: 2: 3(\mathrm{~g})$ (Tan et al. 2011). The SND has been officially enrolled in the Chinese Pharmacopoeia, which has been diffusely used in treatment of cardiovascular diseases for many years (Peter et al. 2013). The SND has been used to be a life-saving drug to treat patients with heart failure (HF), myocardial infarction (MI) and other serious diseases (Hui and Weikang 2001). In the SND, Aconitum carmichaelii, the principal herb, Zingiber officinal, an assistant herb, and Glycyrrhiza uralensis, as a complement and guide herb, work together to get maximal therapeutic efficacy with minimal adverse effects ( $\mathrm{Su}$ et al. 2000). According to the literature, SND can protect against myocardial damage and decreased blood pressure and ease the injuries of hyperlipidemia etc., which contribute remarkable effects to the survival rates of CVD patients (Guo et al. 2008). Regarding the underlying mechanisms and interrelated biological elements of the SND, there are plentiful problems need to be solved imminently: 1) which positive elements take part in the administrative processes of the SND in the treatment of CVDs? 2) Which targets are regulated by the active chemical components to achieve the therapeutic goal? 3) Which pathologic processes are adjusted by the active ingredients and drugs to cure CVDs?. With the great development of system pharmacology today, there are a lot of useful analytical tools to help us understand the complex and whole mechanisms of TCM in treating complex diseases (Zhang et al. 2013). Therefore, in this study, we used a system pharmacology method to dissect clearly molecular mechanisms of the SND in curing CVDs. The Specific research methods can be seen from Figure 1, firstly, the molecular database was constructed and then an in silico ADME system was stuck to filter out the active ingredients with beneficial pharmacokinetics activity. Next, the direct targets of the obtained active compounds are predicted by the in silico model weighted ensemble similarity (WES). Finally, the four networks were constructed to interpret the therapeutic mechanisms of SND for CVDs, which including compound-target-function network, GO analysis, target-CVDs associated disease network and CVDs pathway. The system pharmacology method would provide a novel and efficient way to deeply dissect the chemical and pharmacological basis of SND, which would promote the development of TCMs in the treatment of complex diseases. 


\section{MATERIALS AND METHODS}

In this work, we applied system pharmacology methods to reveal the therapeutic effects of SND (Fig. 1), which comprise: 1) molecular database construction for the 3 drugs in SND; 2) ADME system evaluation to filter potential active components from the above compound database; 3) target-fishing of the obtained potential active components; 4) network construction and analysis to illustrate the underlying molecular mechanisms of SND in curing CVDs.

There are 493 chemical components in SND were collected from literature and database: Traditional Chinese Medicine Systems Pharmacology Database (TCMSP, http://1sp. nwsuaf.edu.cn/tcmsp.php (Ru et al. 2014), TM-MC (Kim et al. 2015) and Phytochemical databases of Chinese herbal constituents (Ehrman et al. 2007). TCMSP include 510 herbal which are registered in Chinese pharmacopoeia with more than 33000 components. It can search the molecular properties, structures, TCM ingredients, targets and diseases handily. TM-MC database offers information on medicinal materials and their chemical compounds from chromatography articles in MEDLINE and PubMed Central. Phytochemical databases of Chinese herbal constituents contain data on known chemical constituents of 240 commonly used Chinese herbs.

\section{EVALUATION OF ADME}

We applied the ADME (absorption, distribution, metabolism, and excretion of drugs) model to filter out the potential active compounds with beneficial pharmacokinetics characteristics. Most of the traditional Chinese medicines are oral preparations. Only through the ADME process and reached the target organs and target tissues can they exert efficacy. ADME predictions could screen out the chemicals which play a vital role. The ADME system comprise predict oral bioavailability
(PreOB), predict drug-likeness (PreDL) and predict Caco-2 permeability (PreCaco-2) models.

PreOB: OB prescreening is one of the significant stages of drugs invention and development, which represents the ratio of oral drugs that reaches the circulation system with their activity unchanged. In this work, a robust in silico model Bioavailable 1.1 (Xu et al. 2012) was utilized to predict the OB value.

PreDL: Drug-likeness is one of the most useful factors in absorption, distribution, metabolism, and elimination for human body. We developed a PreDL model to calculate the DL values by evaluating tanimoto similarity between ingredients and chemicals in the Drug bank database (Wishart et al. 2008).

PreCaco-2: One of the greatest problems for orally administered drugs is its passage through the intestinal epithelial barrier, which decides the rate and proportion of human absorption and ultimately influences its bioavailability (Hilgers et al. 1990). In this work, molecules were chosen as the bioactive ingredients based on standards with $\mathrm{OB} \geq 30 \%$, DL $\geq 0.18$, Caco- $2 \geq 0.4$ for further research.

\section{TARGET FISHING}

In this study, we applied a weighted ensemble similarity (WES) model to predict direct targets of potential active ingredients (Zheng et al. 2015). A system-level identification of drug-target direct interactions is crucial to drug discovery. A novel algorithmtermed weighted ensemble similarity (WES) has been developed to identify drug direct targets based on a large-scale of 98,327 drugtarget relationships. WES model works well in predicting the binding (sensitivity $85 \%$, SEN) and the nonbinding (specificity $71 \%$, SPE) patterns. The accuracy of WES model achieves $78 \%$ and the precision obtains $74 \%$, with the area under the receiver operating curves (AUC) reach's 0.85 . And then, the normalized gene names and 

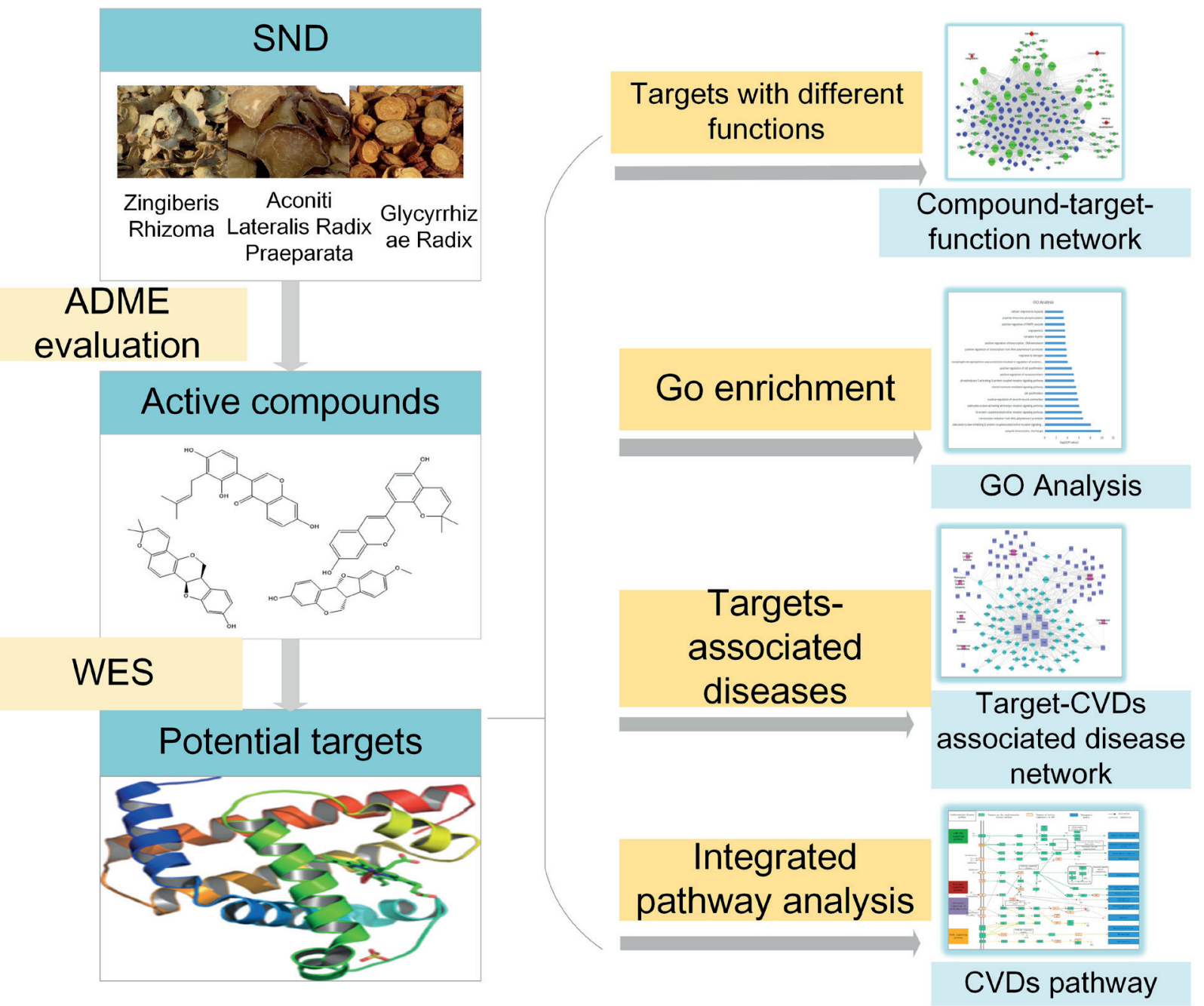

Figure 1 - Worflow for SND in treating CVDs.

organisms of these targets (only Homo sapiens) were obtained from Uniport (http://www.uniprot. org (Magrane and Consortium 2011)) for further analysis. UniProt is used to provide the scientific community with a comprehensive, high-quality and freely accessible resource of protein sequence and functional information.

\section{TARGET FUNCTIONAL ANALYSIS}

To validate whether the 71 selected targets indeed match for CVDs, we performed a Gene Ontology (GO) analysis for their biological process. The 71 potential targets were mapped to DAVID (http:// david.abcc.ncifcrf.gov (Huang et al. 2007)). Then based on the GOBP enrichment data, functional annotation clustering analysis was carried out for further induction of these biological processes into several modules that exert different physiological functions. We only selected the terms with $\mathrm{P}$ value less than 0.01 .

\section{NETWORK CONSTRUCTION}

\section{Compound-target-function network}

A Compound-target-function(C-T-F) network was constructed to explicate the multi-target and multi-function therapeutic feature of the active compounds in treating CVDs. If a protein is 
targeted by a compound, the candidate compound and the potential target protein would be linked. At the same time, targets exert different biological processes were linked to their homologous functional modules.

\section{GO enrichment}

In this part, the GOBP (biological process) enrichment analysis list the top 20 significantly enriched GO terms, which show that the majority of these targets are strongly associated with various biological processes, which mainly including positive regulation of vasoconstriction, phospholipase C-activating G-protein coupled receptor signaling pathway and norepinephrineepinephrine vasoconstriction involved in regulation of systemic arterial blood pressure. These biological processes are all associated with the pathogenesis of CVDs.

\section{Target-disease-category (T-cD) network}

To explore potential targets and diseases interrelationship, the corresponding diseases were extracted from TCMSP and CTD databases (http:// ctdbase.org/ (Mattingly et al. 2006)). Based on the target-associated disease information, a Targetdisease-category (T-cD) network was constructed by linking target with their relevant diseases. The compound-target-function and T-cD networks actualize visualization through Cytoscape 3.4.0. In the network, the compounds, targets, and diseases are represented as nodes, and the interaction between two nodes is represented by an edge. Besides, degree used to evaluate the importance of each node in the networks, which is calculated by Network Analysis plugin of Cytoscape (Shannon et al. 2003).

\section{PATHWAY CONSTRUCTIONS}

According to present cognition of CVDs pathology, we integrate "CVDs pathway" to investigate how targets were influenced by the complex diseases through modulating specific pathways. Firstly, the obtained target proteins were mapped onto KEGG (Ogata et al. 1999) to distribute them to several pathways. Next, pathways closely related to CVDs were picked out and integrated into a "CVDs pathway" based on the pathological and clinical data. Then, a nearness analysis was taken to find the correlativity between "CVDs pathway" proteins and the obtained targets.

\section{RESULTS AND DISCUSSION}

\section{ACTIVE COMPONENTS}

Compounds conform to the inspect criteria and their homologous drugs were taken on the Supplementary Material (Table SI). A total number of 493 compounds were found in the three herbs of SND. With the help of the ADME evaluation system, 78 of active compounds with $\mathrm{OB} \geq 30 \% \quad \mathrm{DL} \geq 0.18, \mathrm{Caco} 2 \geq 0.4$, were obtained and accounting for $15.8 \%$ of all ingredients of the SND. The most of the 78 active ingredients have been proven to that exert vital biological activities including nervous system development, vasoconstriction, transcription, and blood coagulation. For instance, demethylcoclaurine (M05, OB=82.54\%, DL $=0.21$, Caco- $2=0.63$ ) is one of the most effective constituents in ALRP, which plays a protective role on cardiovascular system by enhancing myocardial contractile force and rate. At the same time, it has been reported that demethylcoclaurine increased cardiac output and myocardial oxygen demand and reduced the blood pressure in CVDs (Zhang et al. 2003). Glabridin (M46, OB=53.25, DL=0.47, Caco-2=0.97) could regulate vascular injury and reduce thermogenesis, which is critical to the prevention of atherosclerosis (Fuhrman and Aviram, 2001). As can be seen from Table SII, $\beta$ sitosterol $(\mathrm{M} 07, \mathrm{OB}=36.91, \mathrm{DL}=0.75$, Caco-2=1.32) a common ingredient of 3 drugs in SND, has been demonstrated to significantly 
decrease serum of hyper lipemia mice TC, LDL-C and arterial stiffness index (arteriosclerosis Index), which has good preventive effect on hyperlipidemia and atherosclerosis in mice (Volger et al. 2001).

\section{TARGETS}

Through the methods in 2.3 , we obtained 71 candidate targets for the 78 compounds with 1670 connections between them (Table II). The results are illustrating that most compounds have effect on more than one target, indicating various pharmacological effects of the active molecules.

\section{NETWORK CONSTRUCTION AND ANALYSIS}

\section{Compound-target-function (C-T-F) network}

Figure 2a displays the resultant C-T-F network which is consists of 78 compounds, 71 candidate targets, and 4 corresponding functional annotations. The result displayed an average degree of 22 per compounds and 24 per target proteins, respectively. The relationships between compounds and targets are represented in (Fig. 2b), from those active compounds, M07 exhibits the highest number of target candidate target interaction (degree $=45$ ), followed by M15 (degree = 43), M3 (degree = 40), M44 (degree $=35$ ) and so forth, this gave more knowledge about the multi-target properties of ingredients, which is the innate character of the action mode of herbal drugs. As for the candidate targets, ESR1 (Estrogen receptor, T08) shows the highest degree (Degree $=73$ ), with AR (Androgen receptor, T09, Degree $=71$ ), PTGS2 (Prostaglandin G/H synthase 2, T16, Degree = 69), PPARG (Peroxisome proliferator activated receptor gamma, T12, Degree $=68$ ) behind it, which indicate the potential therapeutic effect of each herb in SND for combating CVDs through the adjustment of these relevant proteins. Many targets are verified in previous research, such as ESR1 (Estrogen receptor), AR (Androgen receptor) and PPAR (Peroxisome proliferator activated receptor gamma). Human coronary arteries shortterm relaxation effects are mostly influenced by the formation of $\mathrm{NO}$, which directly activated by estrogen receptor (Mendelsohn and Karas 1994). Estrogen with physiological concentrations could cause rapid secretion of NO. Thus, the regulation of ESR1 by active ingredients such as M10 and M12 is essential for cardioprotection. Androgen could decrease plasminogen activator and increase coagulation inhibitor and fibrinolysis. Thereby, it preventing thromboembolic disease and myocardial infarction (Tsai et al. 2007). Delton (M2) and Neokadsuranic acid B (M4) could activate AR, which is playing an important role in reducing risk of CVDs (Liu et al. 2003). PPARs has a protective effect on the cardiovascular system, which can be used to prevent and treat heart failure (Finck 2007). Beta-sitosterol (M7) and 7-Methoxy-2-methyl isoflavone (M15) serve as regulator of PPARs which play a role in avoiding heart failure. The functional annotation clustering analysis further summed up relevant biological processes into several functional modules such as blood coagulation, transcription, vasoconstriction and nervous system development $(\mathrm{P}<<0.01)$. For example, (1) ESR2 (Estrogen receptor beta) is active in protection of vascular injury, especially for coronary artery. Clinical data have confirmed that estrogen play an important role in anticoagulation because it can stimulate the production of prostacyclin and nitric oxide and weaken the activation of platelets, which belongs to blood coagulation module (Mahmoodzadeh et al. 2006). (2) The normal level blood pressure is depended on dynamic balance between vasodilation and vasoconstriction. If this balance was broken, it will disturb the normal process of blood fluidity, platelet aggregation, and smooth muscle cell proliferation. Evidence has suggested the clear effect of PTGS2 (Prostaglandin $\mathrm{G} / \mathrm{H}$ synthase 2) in blood pressure regulation, which belongs to the vasoconstriction functional module (Wang et al. 1999). (3) The peroxisome 


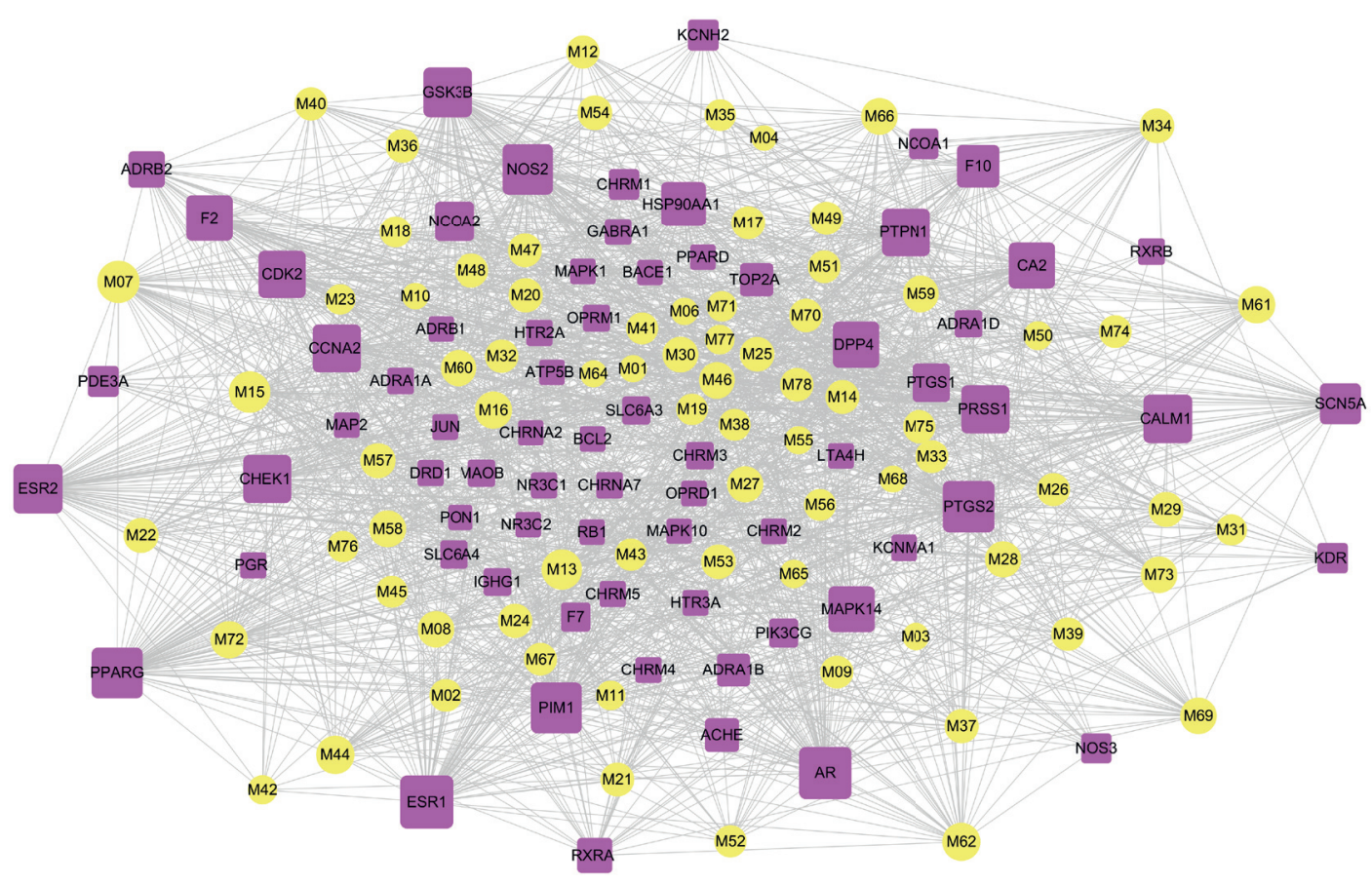

Target

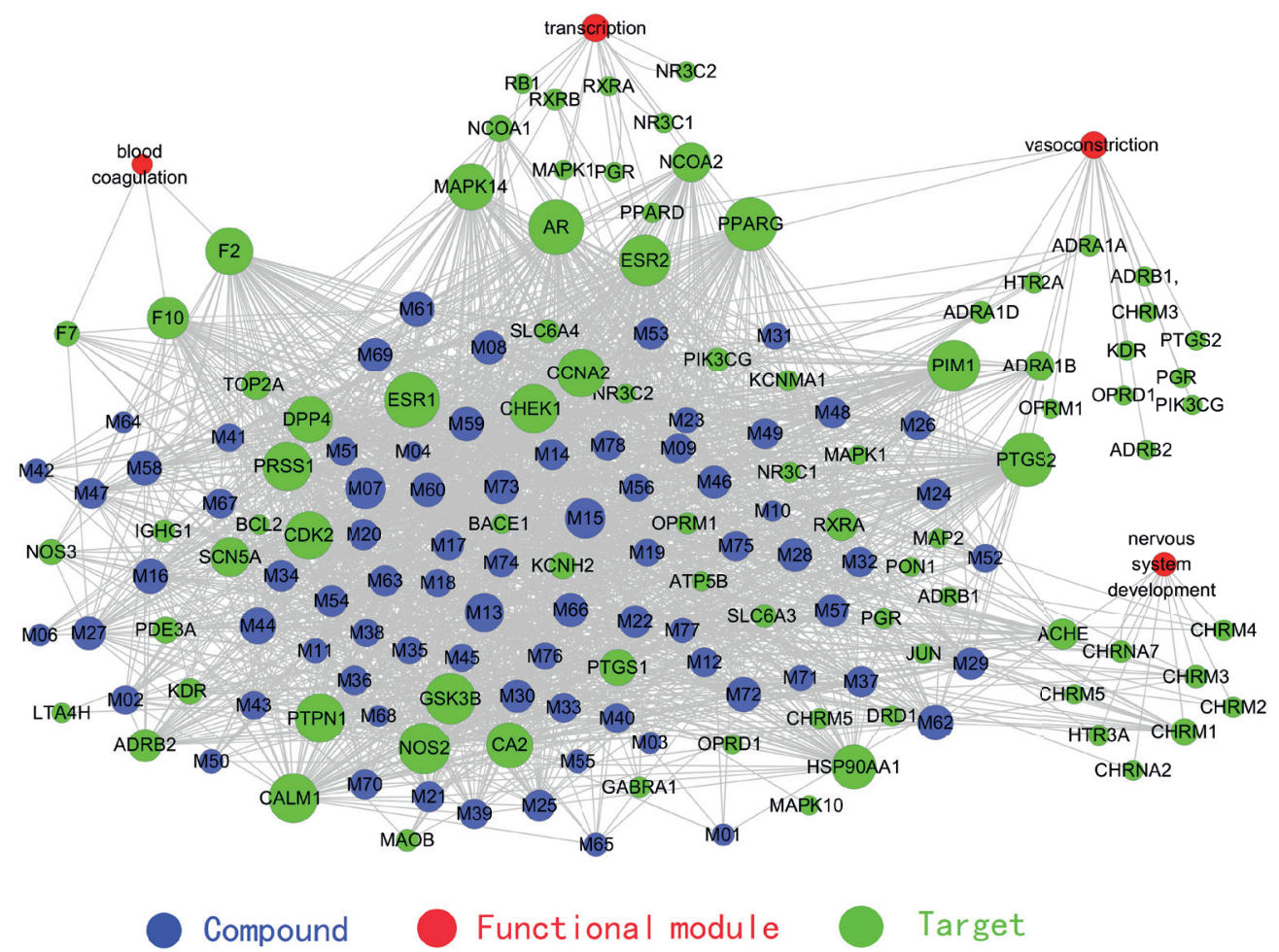

Figure 2 - a. Compound-target-function network. A compound and a target are linked if the target protein is hit by corresponding compound. Similarly, a target and a functional module are linked if the target is involved in this biological process. Node size is proportional to its degree and the letters are node labels. b. Compound-target relationship. A compound and a target are linked if the target protein is hit by the corresponding compound. Node size is proportional to its degree and the letters are node labels. 
proliferator-activated receptors (PPARs) are made up of three related transcription factors that serve to modulate a great number of cellular processes that are vital to cardiovascular health and disease (Cresci 2008). Numerous pharmacologic studies have assessed the effects of specific PPAR agonists in clinical trials, which belong to the transcription functional module. (4) The 5-hydroxytryptamine type-3 (5-HT3) receptor is a cation-selective ion channel of the Cys-loop superfamily. 5-HT3 receptor activation in the central and peripheral nervous systems evokes neuronal excitation and neurotransmitter release, which belongs to the nervous system development functional module (Barnes et al. 2009).

\section{GO enrichment analysis}

As shown in Fig.3, in the GOBP enrichment analysis, we discovered that there are many targets are bound up with several CVDs-associated biological processes, such as synaptic transmission, cholinergic adenylate cyclase-inhibiting, G-protein coupled acetylcholine receptor signaling pathway, transcription initiation from RNA polymerase II promoter, adenylate cyclase-activating adrenergic receptor signaling pathway, positive regulation of smooth muscle contraction and so forth $(\mathrm{P}<<0.01)$, which demonstrate the potential therapeutic effect of each target for CVDs.

\section{Target-CVDs network}

In this part, to show the essence mechanisms of the potential target case by case, relevant CVDs and their associated targets were built onto a target-CVDs (T-D) network. As shown in Fig.4, previous research has confirmed that T10 (ADRB1) and T5 (F2) are closely related to the incidence of myocardial infarction (Yilmaz et al. 2009). Thus, functions of compounds like M15 and M07 are deemed to be a helpful therapeutic plan in myocardial protection from myocardial infarction. As one of the main risk factors of CVDs, hypertension links with more than 30 targets in this T-D network. T06 (KCNH2) plays an important role in regulating the excitatory of smooth muscle cells by potassium channels (Petkov 2012). Besides, T70 (CALM1) is associated with increased risk of sudden cardiac death in patients with chronic heart failure (Liu et al. 2015). All of the above statement demonstrates the multi-target therapeutic efficiency of SND in the treatment of CVDs. At the same time, owing to CVDs are mutual influences exist between different kinds of disease, the shared target proteins of them would be the potentially valuable targets in the treatment of CVDs from an overall view. For example, the adrenal glands receptors play an important role in adjusting heart rate and myocardial contraction force controlled by sympathetic and parasympathetic (Triposkiadis et al. 2009). And lots of studies have demonstrated that in the process of atherosclerosis, T35 (ADRB2) could adjust the diastolic of human heart coronary artery (Milano et al. 1994), which is related to 16 CVDs-associated diseases such as Angina Pectoris, Arrhythmias, Cardiac, Bradycardia, Cardiomegaly, Cardiomyopathies in T-D network. Besides, T11 (SCN5A) gene encodes the forming subunit of the cardiac $\mathrm{Na}+$ channel, which is responsible for the rapid depolarization of the cardiac action potential and plays a role in the control of the excitation and maintenance of the cardiac myocyte excitability (Catterall 2000). A large number of studies have also confirmed that mutations in SCN5A can lead to a variety of fatal familial arrhythmias. Hence, the shared target proteins deserve more attention in subsequent therapy.

\section{PATHWAY ANALYSIS}

According to the present comprehension of CVDs pathology, "CVDs pathway" consists of pathways which are directly related to CVDs. Those target proteins have wondrous functional connection to 


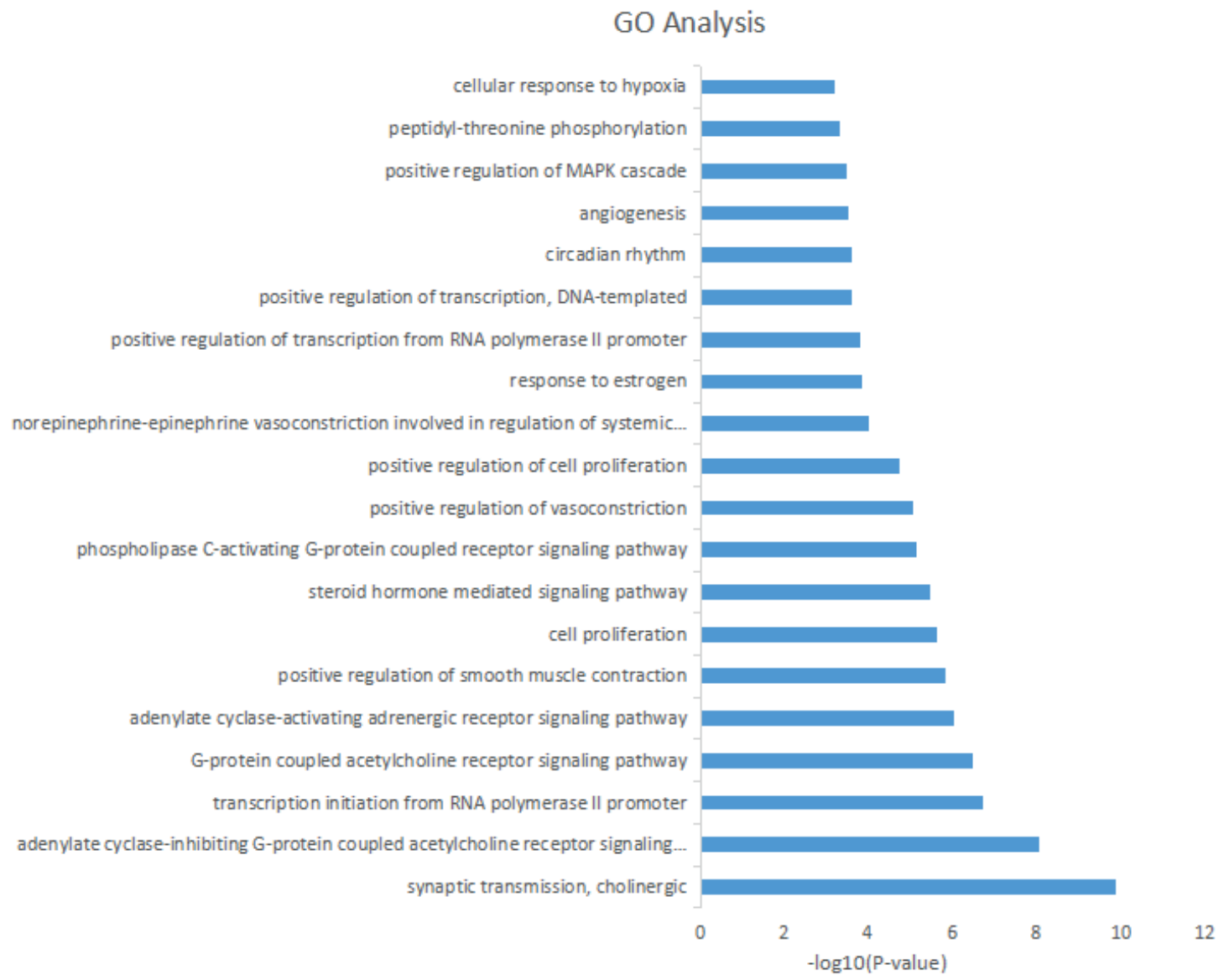

Figure 3 - Gene Ontology (GO)analysis of therapy target genes. They-axis shows significantly enriched 'Biological Process' categories in GO of the target genes, and thex-axis shows the enrichment scores of the $\operatorname{seterms}(\mathrm{P}-$ value $<<0.01)$.

the proteins linked with CVDs pathway. As shown in Fig. 5, we mainly focused on four representative modules to anatomize the underlying therapeutic effects of SND.

\section{CARDIOMYOCYTE PROTECTION MODULE}

A number of experimental evidence suggest that cardiomyocyte apoptosis is one of the major pathogenic factors in heart diseases leading to heart failure (Ganguly et al. 2014). Cardiac hypertrophy is an increase in the muscle volume of the ventricle due to the enlargement of cardiac cells and is also an independent risk factor for cardiovascular diseases (Yamamoto et al. 2011). Therefore, the protection of cardiomyocytes plays a significant role in the prevention and treatment of cardiovascular diseases. Pathways about cardiomyocyte protection operate the function of cardiomyocyte protection through the regulation of some certain active components on their corresponding target proteins. For example, target ERK is marked in cGMP-PKG signaling pathway and target PI3K is marked in erbb signaling pathway. As shown in Figure 5, target genes be marked in the cGMP-PKG signaling pathway are detected to connect with the protection of cardiac myocytes during hypoxia/reoxygenation (Görbe et al. 2007). For instance, M60 (Licochalcone A) was observed to increase ERK (Mitogen-activated protein kinase 1) level, while previous clinical research indicated that ERK activation has an effect 


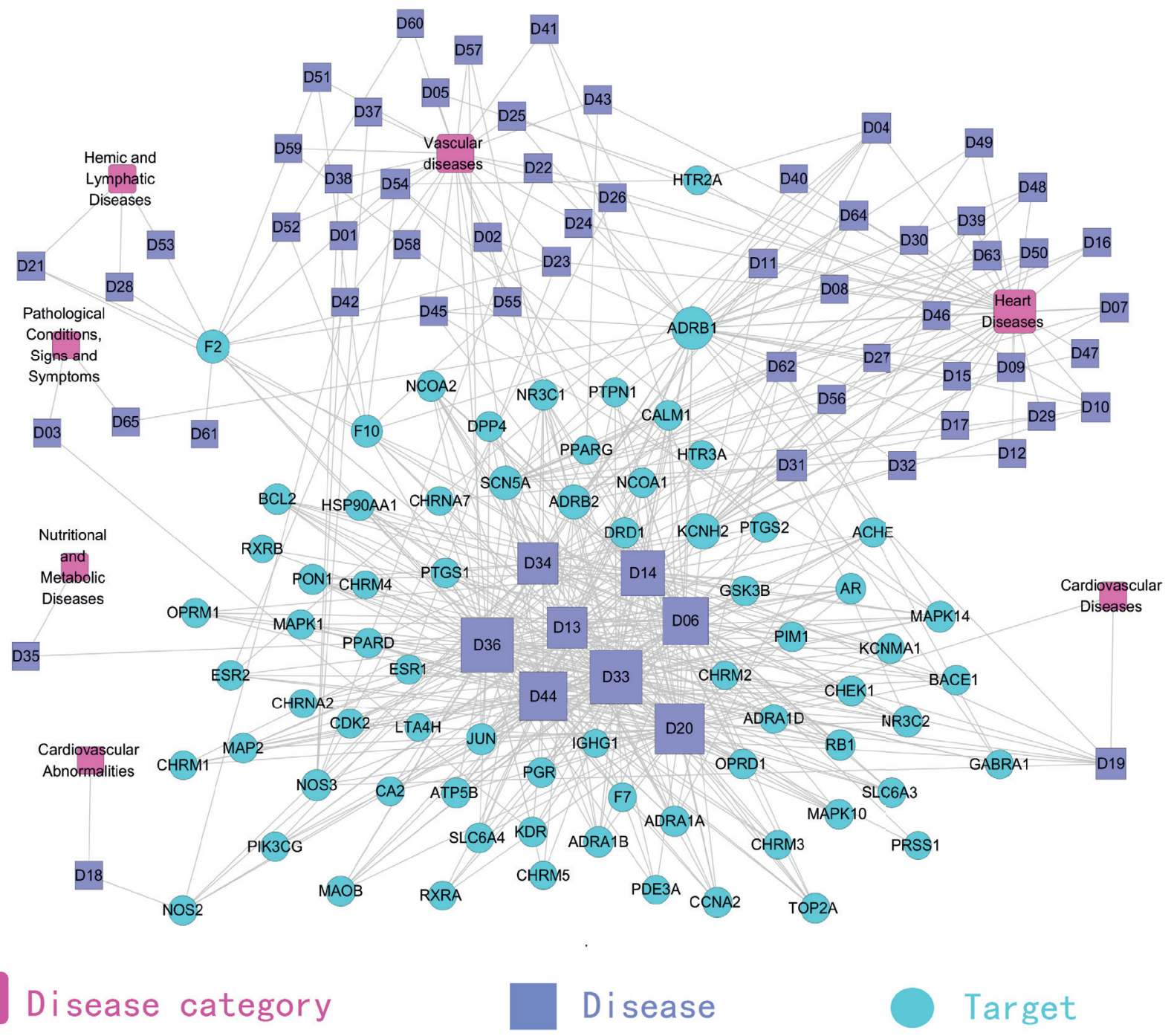

Figure 4 - Target-CVDs associated disease network. Target proteins are linked with their corresponding diseases and those diseases are linked with the corresponding disease categories they belong to. The letters are node labels.

on attenuating oxidative stress induced cardiac apoptosis (Song et al. 2016). And experimental evidence demonstrated that ERK phosphorylation mediates sildenafil-induced myocardial protection against ischemia-reperfusion injury in mice (Das et al. 2009). Besides, ERBB signaling pathway plays an irreplaceable role in regulating cardiomyocyte survival and growth, maintaining cardiomyocyte integrity and intrinsic properties. For example, activation of the NRG1-ERBB4 signaling pathway can repair myocardial cells following myocardial infarction (Liang et al. 2015). 10 molecules were observed to act on the PI3K target in the erbb signaling pathway. Previous study suggested that PI3K target play a key role in cardiomyocyte protection on ERBB signal pathway (Barnes et al. 2009). When cardiomyocytes were damaged, NRG-1 released by endothelial cell could protect the cardiomyocytes from apoptosis due to oxidative overload or anthracycline through inhibiting the release of Cytochrome $\mathrm{C}$ and activating apoptotic protein-3 (Caspase-3) with the PI3K/Akt signaling pathway (Dang et al. 2016). In addition, target GSK3B is regulated by 64 molecules in the erbb 


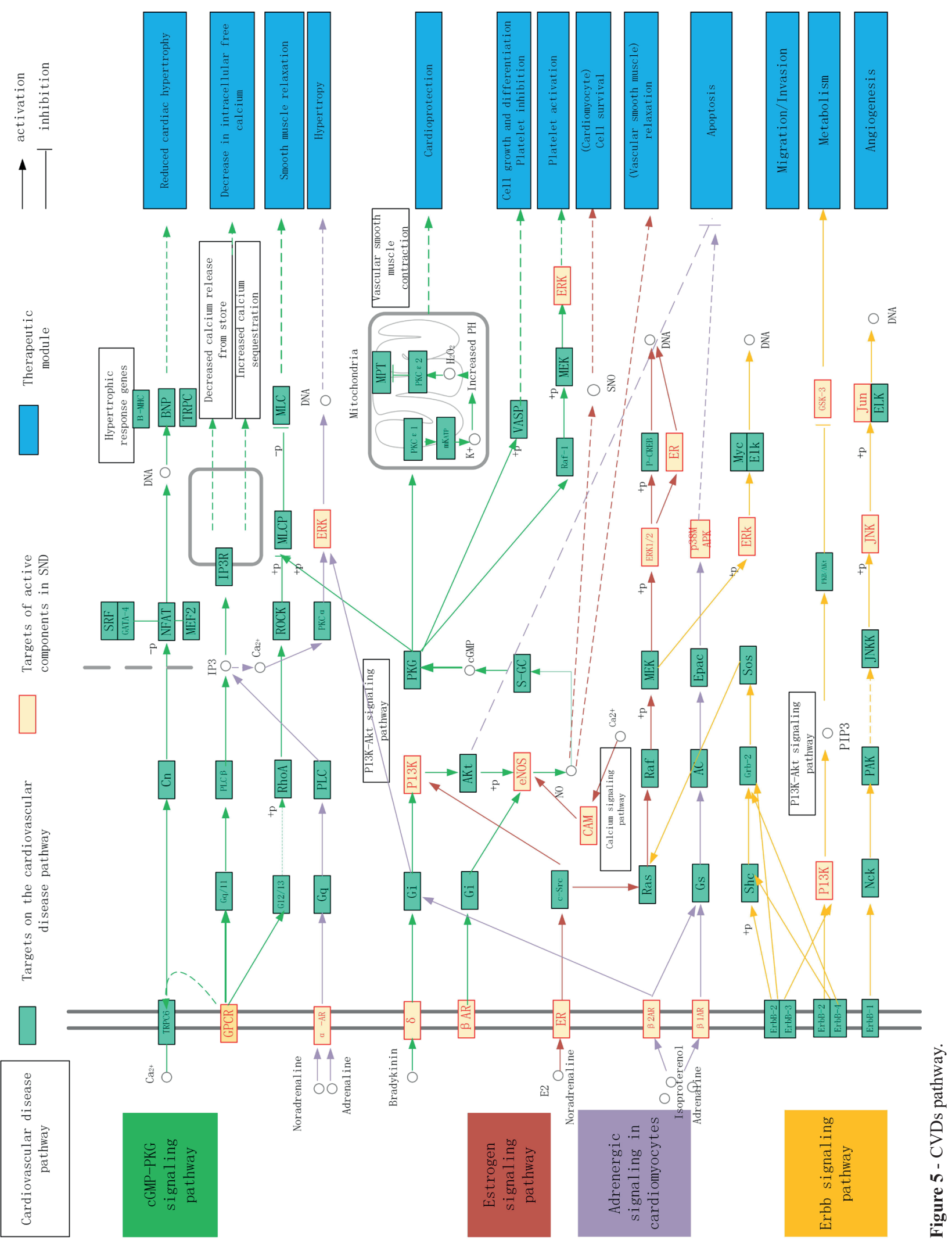


signaling pathway, which mediate myocardial protection by modulation of the mitochondrial permeability transition pore complex. As stated above, SND may cure cardiovascular disease by protecting cardiomyocyte.

\section{BLOOD PRESSURE REGULATION MODULE}

Hypertension is a cardiovascular risk factor present in over two-thirds of people over age 60 in North America; elevated blood pressure correlates with increased risk of heart attack, stroke, and progression to heart and kidney failure. Maintain blood pressure in the normal range is particularly important for the prevention and treatment of cardiovascular disease. As displayed in Fig.5, adrenergic signaling in cardiomyocytes pathway plays an important role in regulating blood pressure. There are 6 molecules act on target ADRA1D (a1-AR) and 4 molecules act on target ADRA1A (a1-AR). Previous study suggested that target a1-AR activates phospholipase C (PLC) through protein $\mathrm{Gq} / 11$ and decomposes PIP2 into inositol triphosphate (IP3) and dieter (DG) on the plasma membrane, which resulting positive changes in myocardium and arterial blood pressure (de Souza et al. 2015). M15 (7-Methoxy-2-methyl isoflavone) were observed to down-regulate the target ADRB1 and studies have shown that downregulation of ADRB1 in myocardial can reduce blood pressure in spontaneously hypertensive rats (Gaiyan and Yuan 2016). There are 29 components acting on target $\beta 2-\mathrm{AR}$, and experimental evidence demonstrated that the haplotype of the ADRB2 receptor is associated with high diastolic blood pressure. Besides, target proteins modulated by active components through operating vascular smooth muscle contraction pathway involve in blood pressure regulation (Qiao et al. 2014). M59 , M61, M62 and M73 were predicted to act on KCNMA1 and previous study suggested that genetic variation of KCNMA1 potassium channel subunits is a risk factor for severe hypertension and myocardial infarction. The components which regulate the target on the VEGF signaling pathway can also inhibit hypertension (Robinson et al. 2010). There are 14 drug components acting on the target KDR, which regulate blood pressure by modulating the expression of nitric oxide synthase (Facemire et al. 2009). All these suggested that the regulation of targets in blood pressure associate pathway is an effective therapeutic mode in treatment of CVDs.

\section{LIPID REGULATION MODULE}

Dyslipidemia as a risk factor for atherosclerosis and coronary heart disease is well established which is defined by abnormal levels of plasma lipoproteins. Active lipid therapy can not only improve vascular endothelial function, stabilize atherosclerotic plaque, delay the process of arteriosclerosis, reduce platelet aggregation, but also significantly reduce the incidence of acute coronary events and the incidence of coronary heart disease and mortality (Kassimatis and Goldsmith 2014). As shown in Fig.5, target ESR1 is modulated by 173 molecules and target ESR2 is regulated by 63 molecules in the estrogen pathway. Target ESR1 and target ESR2 both belong to ER receptor. Previous study showed that activation of ER receptor could reduce the risk of cardiovascular disease by improving dyslipidemia for postmenopausal women (Okamura et al. 2008). Besides, target ERK1/2 can be regulated by M60 (licochalcone a), and previous research suggested that inhibition of ERK1/2 can reduce the ApoE gene-deficient mice atherosclerotic lesions by reversing the transport of cholesterol and inhibiting lipid accumulation (Chen et al. 2015). In addition to estrogen pathway, SND can also regulate the target on PPAR signaling pathway to modulate blood lipids, and then treat cardiovascular diseases (Duval et al. 2007). Target RXRA is modulated by 27 molecules and RXRA polymorphisms in the RXRA and NR1I3 genes could influence lipid 
profile in a Southern Brazilian population (Lima et al. 2013). Thus, all above suggest that SND may treat cardiovascular disease by regulating the lipid regulation module.

\section{CROSS-TALK}

Cross-talk is mean to one or more target genes of one pathway affect(s) another pathway simultaneously. Signaling pathways are not isolated from each other but adjusted the common target to perform different functions. As shown in Fig.5, there exist two typical cross-talks, including PI3K-AKT signaling pathway and Calcium signaling path. PI3K-AKT signaling pathway is a common bond in cGMPPKG signaling pathway, adrenergic signaling in cardiomyocytes, estrogen signaling pathway and erbb signaling pathway. Target PIK3CG is regulated by 10 molecules, which activation of target PIK3CG not only protect cardiomyocytes but also promote blood vessels diastolic, regulate blood pressure. Activation of target PIK3CG can regulate lipid metabolism and reduce the risk of coronary atherosclerosis as well (Xu and Liu 2013). Besides, Calcium signaling pathway is a crosstalk between cGMP-PKG signaling pathway and Estrogen signaling pathway. The main functions of Calcium signaling pathway is to regulate blood pressure. The Calcium channel blockers block calcium influx, reducing intracellular calcium levels, causing vascular smooth muscle relaxation, which were used to treat hypertensive, angina and arrhythmia clinically (Karasawa et al. 1988). There are 13 ingredients act on target eNOS and previous data show that eNOS is important for controlling blood pressure. The fact is reviewed with special emphasis on that endogenous nitric oxide can buffer blood pressure variability (BPV) in dogs, rats and mice (Stauss et al. 2000).

\section{CONCLUSIONS}

We applied a systems pharmacology approach by integrating the ADME screening, targets fishing, network construction, and analysis to dissect the underlying mechanisms of action of SND in treating of CVDs. The main findings are as follows. 78 molecules and 71 direct targets were obtained, which suggested that a multi-drug-multi-target paradigm of SND. These compounds and targets might serve to guide the further study of SND. The target and C-T-F network analysis together display that some vital compounds of SND such as demethylcoclaurine, glabridin and $\beta$ sitosterol which may play vital role in treatment of CVDs. SND positively aiming for some targets like ESR1, AR, and PTGS2 exhibits the therapeutic effects against CVDs by modulating blood coagulation, transcription, vasoconstriction, and nervous system development function. The target-CVDs network and CVDs pathway display that SND might exert synergistic effect with multi-drug-multi-disease interaction in treatment of CVDs. Different targets act on the same pathways or multiple pathways influenced by the same targets get their more chances to affecting the whole equilibrium of networks, which make the SND therapy more effective. SND exert its excellent efficacy in the fighting with CVDs from an overall standpoint with the regulation of cardiomyocyte protection module, blood pressure regulation module, and lipid regulation module. This work provides a novel approach for comprehension of the mechanism of SND on CVDs from molecular level to pathway level. The results are also facilitating the wide spread application of traditional medicines in modern medicine.

\section{ACKNOWLEDGMENTS}

All the authors of the manuscript are immensely grateful to their respective universities and institutes for their technical assistance and valuable support 
in the completion of this research project. This work was supported by Grants from Northwest A \& F University, National Natural Science Foundation of China (31170796 and 81373892, respectively). It was also supported in part by Grants from National Natural Science Foundation of China (31540008). We report that there's no conflict of interest.

\section{AUTHOR CONTRIBUTIONS}

Zhiming Shu, Tiantian Wu, Mohamed Shahen, Zihu Guo, Jia Shu, He Wang, Akhtar Hussain Shar, and Chaobin Liu designed the study, collected literature, performed the experiment, analyzed the data, wrote the paper, and drafted the manuscript. Mayada Ragab Farag, Mahmoud Alagawany and Mohamed Ezzat Abd El-Hack reviewed the manuscript and performed the final check. All authors have read and approved the final manuscript.

\section{REFERENCES}

BARNES NM, HALES TG, LUMMIS SCR AND PETERS JA. 2009. The 5-HT 3 receptor - the relationship between structure and function. Neuropharmacol 56(1): 273.

CATTERALL WA. 2000. From ionic currents to molecular mechanisms: the structure and function of voltage-gated sodium channels. Neuron 26(1): 13-25.

CHEN Y, DUAN Y, YANG X, SUN L, LIU M, WANG Q, MA X, ZHANG W, LI X AND HU W. 2015. Inhibition of ERK1/2 and activation of LXR synergistically reduce atherosclerotic lesions in ApoE-deficient mice. Arterioscler Thromb Vasc Biol 35(4): 948-959.

CRESCI S. 2008. PPAR Genomics and Pharmacogenomics: Implications for Cardiovascular Disease. Ppar Re 2008(12): 374549.

DANG R, GUO Y, CAI H, YANG R, LIANG D, LV C AND JIANG P. 2016. Effects of prolonged antipsychotic administration on neuregulin-1/ErbB signaling in rat prefrontal cortex and myocardium: implications for the therapeutic action and cardiac adverse effect. J Toxicol Sci 41(2): 303-309.

DAS A, SALLOUM FN, XI L, RAO YJ AND KUKREJA RC. 2009. ERK phosphorylation mediates sildenafil-induced myocardial protection against ischemia-reperfusion injury in mice. Am J Physiol-Heart Circulat Physiol 296(5): H1236-H1243.

DE SOUZA AA, DE MENEZES RC, ABREU AR, ARAUJO GR, COSTA DC AND CHIANCA DA. 2015. Increased $\alpha 1$-adrenoreceptor activity is required to sustain blood pressure in female rats under food restriction. Life Sci 128: 55-63.

DUVAL C, MÜLLER M AND KERSTEN S. 2007. PPAR and dyslipidemia. Biochimica et Biophysica Acta (BBA)Mole Cell Biol Lipids 1771(8): 961-971.

EHRMAN TM, BARLOW DJ AND HYLAND PJ. 2007. Phytochemical databases of Chinese herbal constituents and bioactive plant compounds with known target specificities. J Chem Info Mod 47(2): 254.

FACEMIRE C, NIXON AB, GRIFFITHS R, HURWITZ H AND COFFMAN TM. 2009. Vascular endothelial growth factor receptor 2 controls blood pressure by regulating nitric oxide synthase expression. Hypertension 54(3): 652658.

FINCK BN. 2007. The PPAR regulatory system in cardiac physiology and disease. Cardiovascular Res 73(2): 269277.

FUHRMAN B AND AVIRAM M. 2001. Flavonoids protect LDL from oxidation and attenuate atherosclerosis. Curr Opin Lipidol 12(1): 41-48.

GÖRBE A, GIRICZ Z, HULIÁK I, BAXTER G AND FERDINANDY P. 2007. NO-cGMP-PKG signalling pathway involved in the protection of cardiac myocytes during hypoxia/reoxygenation. J Mole Cell Cardiol 42(6): S48-S49.

GAIYAN W AND YUAN H. 2016. GW27-e0227 Downregulation of the [beta] 1 adrenergic receptor in myocardium by AAV9-shRNA-ADRB1-ZsGreen injection reduce blood pressure in spontaneously hypertensive rats. J Am Coll Cardiol 68(16): C7.

GANGULY S, MITRA A AND SARKAR S. 2014. Role of $\alpha$-Crystallin B in Regulation of Stress Induced Cardiomyocyte Apoptosis. Cardiovascular Hematoll Agents Med Chem 12(2): 60-65.

GUO Y-L, YANG X-W, XIAO S-Y, CHONG Z AND LIN W-H. 2008. The regulating effect and mechanism of Sini decoction on vascular active substance in renovascular hypertensive rats. Chin Pharmacol Bull 24(1): 79.

HILGERS AR, CONRADI RA AND BURTON PS. 1990. Caco-2 cell monolayers as a model for drug transport across the intestinal mucosa. Pharmaceut Res 7(9): 902910.

HUANG DW, SHERMAN BT, TAN Q, KIR J, LIU D, BRYANT D, GUO Y, STEPHENS R, BASELER MW AND LANE HC. 2007. DAVID Bioinformatics Resources: expanded annotation database and novel algorithms to better extract biology from large gene lists. Nucl Acids Res 35(suppl 2): W169-W175.

HUI Y AND WEIKANG W. 2001. An Experimental Study on the Influence of Sini Tang as a Whole and Its Ingredients on Hemodynamics Rats With Heart Failure [J]. New J Trad Chin Med 11: 053. 
KARASAWA A, KUBO K, SHUTO K, OKA T AND NAKAMIZO N. 1988. Antihypertensive effects of the new calcium antagonist benidipine hydrochloride in rats. Arzneimittelforschung 38(11A): 1684-1690.

KASSIMATIS T AND GOLDSMITH D. 2014. CVD in CKD: Focus on the Dyslipidemia Problem. Springer New York.

KHAND A, RANKIN A, KAYE G AND CLELAND J. 2000. Systematic review of the management of atrial fibrillation in patients with heart failure. Eur Heart J 21(8): 614-632.

KIM SK, NAM SJ, JANG H, KIM A AND LEE JJ. 2015. TM-MC: a database of medicinal materials and chemical compounds in Northeast Asian traditional medicine. BMC Compl Alternt Med 15(1): 218.

LIANG X, DING Y, ZHANG Y, CHAI Y, HE J, CHIU S, GAO F, TSE H AND LIAN Q. 2015. Activation of NRG1-ERBB4 signaling potentiates mesenchymal stem cell-mediated myocardial repairs following myocardial infarction. Cell Death Disease 6(5): e1765.

LIMA LO, BRUXEL EM, HUTZ MH, VAN DER SAND CR, VAN DER SAND LC, FERREIRA MEW, PIRES RC, FIEGENBAUM M AND ALMEIDA S. 2013. Influence of PPARA, RXRA, NR1I2 and NR1I3 gene polymorphisms on the lipid-lowering efficacy and safety of statin therapy. Arquivos Brasileiros de Endocrinologia Metabologia 57(7): 513-519.

LIU PY, DEATH AK AND HANDELSMAN DJ. 2003. Androgens and cardiovascular disease. Endocrine reviews 24(3): 313-340.

LIU Z, LIU X, YU H, PEI J, ZHANG Y, GONG J AND PU J. 2015. Common Variants in TRDN and CALM1 Are Associated with Risk of Sudden Cardiac Death in Chronic Heart Failure Patients in Chinese Han Population. Plos One 10(7): e0132459.

MAGRANE M AND CONSORTIUM U. 2011. UniProt Knowledgebase: a hub of integrated protein data. Database 2011, bar009.

MAHMOODZADEH S, EDER S, NORDMEYER J, EHLER E, HUBER O, MARTUS P, WEISKE J, PREGLA R, HETZER R AND REGITZ-ZAGROSEK V. 2006. Estrogen receptor alpha up-regulation and redistribution in human heart failure. FASEB J 20(7): 926-934.

MATTINGLY C, ROSENSTEIN M, COLBY G, FORREST JR J AND BOYER J. 2006. The Comparative Toxicogenomics Database (CTD): a resource for comparative toxicological studies. J Expl Zool A: Ecolog Gen Physiol 305(9): 689692.

MENDELSOHN ME AND KARAS RH. 1994. Estrogen and the blood vessel wall. Curr Opin Cardiol 9(5): 619-626.

MILANO C, ALLEN L, ROCKMAN H, DOLBER P, MCMINN T, CHIEN K, JOHNSON T, BOND R AND LEFKOWITZ R. 1994. Enhanced myocardial function in transgenic mice overexpressing the beta-2-adrenergic receptor. Science 264(5158): 582-587.
OGATA GOT, SATO K, FUJIBUCHI W, BONO H AND KANEHISA M. 1999. KEGG: Kyoto encyclopedia of genes and genomes. Nucl Acid Res 27(1): 29-34.

OKAMURA S, SAWADA Y, SATOH T, SAKAMOTO H, SAITO Y, SUMINO H, TAKIZAWA T, KOGURE T, CHAICHANTIPYUTH C AND HIGUCHI Y. 2008. Pueraria mirifica phytoestrogens improve dyslipidemia in postmenopausal women probably by activating estrogen receptor subtypes. Tohoku J Exp Med 216(4): 341-351.

PETER K, SCHINNERL J, FELSINGER S, BRECKER L, BAUER R, BREITENEDER H, XU R AND MA Y. 2013. A novel concept for detoxification: complexation between aconitine and liquiritin in a Chinese herbal formula ('Sini Tang'). J Ethnopharmacol 149(2): 562-569.

PETKOV GV. 2012. Role of potassium ion channels in detrusor smooth muscle function and dysfunction. Nature Rev Urol 9(1): 30-40.

QIAO Y-N, HE W-Q, CHEN C-P, ZHANG C-H, ZHAO W, WANG P, ZHANG L, WU Y-Z, YANG X AND PENG Y-J. 2014. Myosin phosphatase target subunit 1 (MYPT1) regulates the contraction and relaxation of vascular smooth muscle and maintains blood pressure. J Biol Chem 289(32): 22512-22523.

ROBINSON ES, KHANKIN EV, KARUMANCHI SA AND HUMPHREYS BD. 2010. Hypertension induced by VEGF signaling pathway inhibition: mechanisms and potential use as a biomarker. Seminars Nephrol 30(6): 591-601.

RU J, LI P, WANG J, ZHOU W, LI B, HUANG C, LI P, GUO Z, TAO W AND YANG Y. 2014. TCMSP: a database of systems pharmacology for drug discovery from herbal medicines. J Cheminformat 6(1): 13.

RUSSELL RP. 1988. Side effects of calcium channel blockers. Hypertension 11(3 Pt 2): II42.

SHANNON P, MARKIEL A, OZIER O, BALIGA NS, WANG JT, RAMAGE D, AMIN N, SCHWIKOWSKI B AND IDEKER T. 2003. Cytoscape: a software environment for integrated models of biomolecular interaction networks. Genome Res 13(11): 2498-2504.

SHU Z ET AL. 2018. Clarifying the mechanism of Sinisan formula for treatment of chronic hepatitis by systems pharmacology method. Biomed Pharmacother 100: 532550.

SONG Y-H, CAI H, ZHAO Z-M, CHANG W-J, GU N, CAO S-P AND WU M-L. 2016. Icariin attenuated oxidative stress induced-cardiac apoptosis by mitochondria protection and ERK activation. Biomed Pharmacother 83: 1089-1094.

STAUSS HM, NAFZ B, MROWKA R AND PERSSON PB. 2000. Blood pressure control in eNOS knock-out mice: comparison with other species under NO blockade. Acta Physiol 168(1): 155-160.

SU J, LIN S, CHEN L AND WU W. 2000. Study on influence of Sini Decoction on quality of life of patients after 
percutaneous transluminal coronary angioplastyon quality of life of patients after percutaneous transluminal coronary angioplasty. Chin J Integr Med 6(2): 108-111.

SU S-L, DUAN J-A, ZHAO X-H, HUA Y-Q, HOU P-F, SHANG E-X, TANG Y-P AND DING A-W. 2008. Bioactive components from oils of Siwu Decoction and Xiangfu Siwu Decoction by gas chromatography: mass spectrometry and principle components analysis. World Sci Technol 10(2): 50-57.

TAN G, ZHU Z, JING J, LV L, LOU Z, ZHANG G AND CHAI Y. 2011. Characterization of constituents in Sini decoction and rat plasma by high-performance liquid chromatography with diode array detection coupled to time-of-flight mass spectrometry. Biomedl Chromatograph 25(8): 913-924.

TRIPOSKIADIS F, KARAYANNIS G, GIAMOUZIS G, SKOULARIGIS J, LOURIDAS G AND BUTLER J. 2009. The sympathetic nervous system in heart failure: physiology, pathophysiology, and clinical implications. J Am Coll Cardiol 54(19): 1747-1762.

TSAI HK, D'AMICO AV, SADETSKY N, CHEN M-H AND CARROLL PR. 2007. Androgen deprivation therapy for localized prostate cancer and the risk of cardiovascular mortality. J National Cancer Institute 99: 1516-1524.

VOLGER O, MENSINK R, PLAT J, HORNSTRA G, HAVEKES L AND PRINCEN H. 2001. Dietary vegetable oil and wood derived plant stanol esters reduce atherosclerotic lesion size and severity in apoE* 3-Leiden transgenic mice. Atherosclerosis 157: 375-381.

WANG J-L, CHENG H-F AND HARRIS RC. 1999. Cyclooxygenase-2 inhibition decreases renin content and lowers blood pressure in a model of renovascular hypertension. Hypertension 34: 96-101.

WISHART DS, KNOX C, GUO AC, CHENG D, SHRIVASTAVA S, TZUR D, GAUTAM B AND HASSANALI M. 2008. DrugBank: a knowledgebase for drugs, drug actions and drug targets. Nucleic Acids Res 36(Suppl 1): D901-D906.

XU LAND LIU Y. 2013. Administration of telmisartan reduced systolic blood pressure and oxidative stress probably through the activation of PI3K/Akt/eNOS pathway and NO release in spontaneously hypertensive rats. Physiol Res 62(4): 351.
XU X, ZHANG W, HUANG C, LI Y, YU H, WANG Y, DUAN J AND LING Y. 2012. A novel chemometric method for the prediction of human oral bioavailability. Int J Mole Sci 13: 6964-6982.

YAMAMOTO S, KITA S, IYODA T, YAMADA T AND IWAMOTO T. 2011. New molecular mechanisms for cardiovascular disease: cardiac hypertrophy and cellvolume regulation. J Pharmacol Sci 116: 343-349.

YATOO MI ET AL. 2017. Beneficial health applications and medicinal values of Pedicularis plants: A review. Biomed Pharmacother 95: 1301-1313.

YILMAZ A, KAYA MG, MERDANOGLU U, ERGUN MA, CENGEL A AND MENEVSE S. 2009. Association of $\beta-1$ and $\beta-2$ adrenergic receptor gene polymorphisms with myocardial infarction. J Clin Lib Anal 23: 237-243.

ZHANG W, HUAI Y, MIAO Z, CHU C, SHAHEN M, UR RAHMAN S, ALAGAWANY M, ABD EL-HACK ME, ZHAO H AND QIAN A. 2019. Systems pharmacology approach to investigate the molecular mechanisms of herb Rhodiola rosea L. radix. Drug Devel Indust Pharm 45: 456-464.

ZHANG B, WANG X AND LI S. 2013. An integrative platform of TCM network pharmacology and its application on a herbal formula, Qing-Luo-Yin. Evid Based Compl. Alternat Med 2013.

ZHANG Z, TAO Z, SHI R, ZHANG X, LIU Y, HE Z-X, CHEN B AND LIU X. 2003. Measurement of the effects and safety of Higenamine, a Chinese herbal medicine on cardiovascular system: Could it represent a new pharmacological myocardial stress agent? World J Nucl Med 2: 287-291.

ZHENG C, GUO Z, HUANG C, WU Z, LI Y, CHEN X, FU Y, RU J, SHAR PA AND WANG Y. 2015. Large-scale direct targeting for drug repositioning and discovery. Sci Rep 5: 11970.

\section{SUPPLEMENTARY MATERIAL}

Table SI - Components of drugs in SND.

Table SII - Targets of SND. 